\title{
Dopamine D2 Modulation of Sign and Goal Tracking in Rats
}

\author{
Juan Carlos Lopez ${ }^{1,2}$, Rose-Marie Karlsson ${ }^{2}$ and Patricio O'Donnell*,2,3,4 \\ 'Departamento de Psicología Experimental, Universidad de Sevilla, Sevilla, Spain; '2Department of Anatomy and Neurobiology, University of \\ Maryland School of Medicine, Baltimore, MD, USA; ${ }^{3}$ Department of Psychiatry, University of Maryland School of Medicine, Baltimore, MD, USA
}

\begin{abstract}
In Pavlovian conditioning, sign- and goal-tracking behaviors represent different approaches towards the conditioned stimulus. These behavioral patterns have been associated with predictive or incentive properties of the conditioned stimulus, with a crucial involvement of the mesolimbic dopamine system. As it is possible that sign tracking behavior is more sensitive to dopamine modulation, we evaluated the dopamine-dependence of sign- and goal-tracking behavior. We assessed responses to both a D2 agonist and an antagonist, and tested performance in a behavioral paradigm known to activate dopamine projections and in an animal model that affects mesolimbic and mesocortical function. Sign trackers displayed a greater sensitivity to a D2 agonist and smaller prepulse inhibition of the acoustic startle response than goal trackers, suggesting a reduced inhibitory ability. In addition, a neonatal ventral hippocampal lesion resulted in the loss of incentive salience of cues in sign trackers. Overall, these data indicate that sign-tracking behavior is more heavily controlled by dopamine than goal tracking.

Neuropsychopharmacology (20I5) 40, 2096-2102; doi:I0.1038/npp.20I5.68; published online I April 2015
\end{abstract}

\section{INTRODUCTION}

How external stimuli are processed in the rodent brain can be assessed using reward-associated cues. During Pavlovian conditioning, pairing of conditioned stimulus (CS) with reward, the unconditioned stimulus (US), leads to conditioned reward-approach responses. conditioned reward behaviors can be sign-tracking (ST), in which the animal engages with the CS, or goal-tracking (GT), in which the animals approach the location of the reward (Flagel et al, 2007; Robinson and Flagel, 2009). For ST animals, the CS itself may have incentive value, acting as a surrogate of the US. For GT rats, on the other hand, the CS is only a signal predictive of future reward delivery (Robinson and Flagel, 2009; Flagel et al, 2011).

The mesolimbic dopamine system is involved in processing both predictive and incentive stimuli and may play different roles in ST and GT approaches to reward-predicting stimuli. Indeed, ST and GT rats express different patterns of dopamine release in the nucleus accumbens (NA) (Flagel et al, 2011). A CS increased NA dopamine in ST, but not GT rats, consistent with the notion of mesolimbic dopamine being related to the incentive salience of reward and eventually allowing signals associated with reward to become attractors (Berridge and Robinson, 1998; Berridge, 2007). It is therefore possible that dopamine plays out more strongly in ST rats when a discrete cue is used as CS, and altering

* Correspondence: Professor P O'Donnell, Neuroscience Research Unit Pfizer Inc., 610 Main St, Cambridge, MA 02139, USA, Tel: + I 617395 0838, Fax: + I 845474 4276, E-mail: patricio.odonnell@pfizer.com ${ }^{4}$ Current Address: Neuroscience Research Unit, Pfizer, Inc.

Received 9 November 2014; revised 9 February 2015; accepted 13 February 2015; accepted article preview online II March 2015 dopamine neurotransmission may affect ST to a greater extent than GT behaviors. Here, we tested this possibility by assessing ST and GT behavior in rats and by manipulating dopamine transmission by administration of either D2 agonist or antagonist. Furthermore, we assessed sensory processing in ST and GT rats using the prepulse inhibition of the acoustic startle response (PPI), a phenomenon dependent on D2 receptor function (Swerdlow et al, 2001). Lastly, we assessed whether a neonatal ventral hippocampal lesion (NVHL), a developmental manipulation that affects dopamine modulation of corticolimbic circuits (Goto and O'Donnell, 2002), affected GT and ST behaviors and their dopamine modulation.

\section{MATERIALS AND METHODS}

\section{Animals}

Experimentally naive adult male Long Evans rats (200-400 g) were obtained from Charles River (Wilmington, MA) and housed in groups of three, with food and water freely available, in temperature- and humidity-controlled rooms and kept on a 12-h inverted light cycle (lights off at 8:00 AM). After arrival, rats were given 1 week to acclimate to the colony room before testing began. All rats were handled daily in their home cages. Timed pregnant Long-Evans rats were obtained at gestational days 13-15 from Charles River. Between postnatal day 7 and 8, male pups (15-20 g) received either an excitotoxic lesion of the ventral hippocampus or sham procedure as previously described (Chambers and Lipska, 2011). In short, pups were anesthetized with hypothermia and secured to a Styrofoam platform attached to a stereotaxic frame (David Kopf Instruments, Tujunga, CA). NVHL rats received a bilateral infusion of $0.3 \mu \mathrm{l}$ per 
side of ibotenic acid $(10 \mu \mathrm{g} / \mu \mathrm{l}$ in artificial cerebrospinal fluid; ToCris, Minneapolis, MN) into the ventral hippocampus ( $3 \mathrm{~mm}$ rostral to bregma; $3.5 \mathrm{~mm}$ lateral to midline; and $5 \mathrm{~mm}$ from surface) at a rate of $0.15 \mu \mathrm{l} / \mathrm{min}$. Sham surgeries were carried out in exactly the same manner but without any liquid infusion. After the procedure, wounds were clipped and when pups' activity levels had returned to normal, they were returned to their dams and remained undisturbed, except for husbandry, until the wound clips were removed and animals weaned at postnatal day 23. Twenty-one adult rats that had received a NVHL or sham surgery were tested for GT and ST profiles as described above. All experiments were run during the active, dark phase between 10.00 and $16.00 \mathrm{~h}$. All procedures were conducted according to the USPHS 'Guide for the care and use of laboratory animals' and approved by the University of Maryland School of Medicine Institutional Animal Care and Use Committee.

\section{Behavioral Testing}

Rats were tested in eight operant conditioning chambers (Med Associates, St Albans, VT) housed within soundattenuating enclosures. Each chamber was equipped with a food receptacle in the center of one wall with one illuminated retractable lever (located $9 \mathrm{~cm}$ above the floor and $2.5 \mathrm{~cm}$ to the left or right of the food receptacle). Lever location was counterbalanced across rats. The wall opposite the food cup contained a house light that remained illuminated throughout the session and a nose poke hole to analyze the activity and exploratory level in the different groups. A ventilating fan provided background noise. Head entries into the food cup or nose poke hole were recorded by beam breaks of an infrared photobeam located inside. Lever presses and beam breaks were recorded using MED-PC software (Med Associates), and only the first event after the cue in any trial was recorded.

Pre-training. The procedures used for behavioral testing were similar to those described previously (Flagel et al, 2007). Two days before the start of Pavlovian conditioning, $45 \mathrm{mg}$ chocolate-flavored food pellets (TestDiet) were placed into the home cages to familiarize the rats with the food. During the next stage (pre-training sessions), each rat was tested under white noise with lever retracted. Twenty-five food pellets were delivered on a variable interval 90 -s schedule (60 s the lowest intertrial interval and $120 \mathrm{~s}$ the highest). At the end of each session, it was checked whether rats had obtained all the pellets from the magazine.

Pavlovian conditioning. This phase consisted of four consecutive sessions, with 25 trials per session. Each individual trial consisted of presentation of the illuminated lever (CS) into the chamber for $8 \mathrm{~s}$, and after the retraction of the lever press, the pellet dispenser was activated and one food pellet (US) was delivered into the food receptacle. CSUS pairings were programmed on a 60 -s variable interval schedule. The following measures were recorded during this period: number of lever presses, number of receptacle entries during presentation of the CS, number of nose-pokes, and latencies to the first lever press and the first receptacle entry. After the third session, rats were separated in two groups according to whether they reached criteria for GT or ST. Rats were classified as ST or GT using the Pavlovian Conditioned Approach index (Meyer et al, 2012). According to this index, ST scores were higher than 0.59 , and GT lower than -0.53 .

Prepulse inhibition of the acoustic startle response (PPI). Another cohort of 22 rats was used to test for PPI after session four. PPI was tested in four chambers designed to detect and record startle responses in rodents (San Diego Instruments, San Diego, CA). The chambers had a background white noise $(70 \mathrm{~dB})$, and the PPI session consisted of trials with startling pulse $(120 \mathrm{~dB}, 100 \mathrm{~ms})$, this pulse preceded by prepulses of three different intensities $(5,10$, and $15 \mathrm{~dB}$ above background; $120 \mathrm{~ms}$ prior to pulse), null trials, and some trials with prepulse alone. Inter-trial intervals were $5-15 \mathrm{~s}$.

\section{Drug Administration}

Eighteen rats were used to test the effect of D2 pharmacology on ST and GT behavior. The selective D2 antagonist S(-) eticlopride hydrochloride $(20 \mathrm{ng} / \mathrm{kg}$, i.p.; Sigma, St Louis, $\mathrm{MO}$ ) and the D2 agonist (-)quinpirole hydrochloride (200 ng/kg, i.p., Sigma) were dissolved in $0.9 \%$ saline and administered $20 \mathrm{~min}$ prior to test sessions in a volume of $0.5 \mathrm{ml} / \mathrm{kg}$ prior to the 5 th, 6 th, and 7 th sessions. Drug administration in NVHL rats was also performed after training. Because of different sensitivity to drugs, two doses of S( - )eticlopride hydrochloride (10 and $20 \mathrm{ng} / \mathrm{kg}$, i.p.) were administered to GT animals. ST rats received ( - )quinpirole hydrochloride (100 ng/kg and $200 \mathrm{ng} / \mathrm{kg}$ i.p.) after session 3.

\section{Histology}

Upon completion of behavioral testing of NVHL rats, each rat was deeply anesthetized with chloral hydrate $(400 \mathrm{mg} / \mathrm{kg}$, i.p.) and perfused transcardially with a fixative solution $(10 \%$ formalin in phosphate buffer $0.1 \mathrm{M}, \mathrm{pH}$ 7.4). The brains were removed from the skull and placed in $10 \%$ formalin and buffer for 3-4 days. Next, the brains were cut with a microtome in the coronal plane at $50 \mu \mathrm{m}$ thickness and Nisslstained for histological analysis of hippocampus damage. This method allowed verifying the cell loss, thinning, gliosis, cellular disorganization, and enlarged ventricles. An observer blind to the lesion status analyzed the lesion extent. Only rats with evidence of bilateral ventral hippocampal damage and without significant damage to adjacent structures were included in this study.

\section{Statistical Analyses}

SPSS statistical software was used to analyze the results of the experiments. We used repeated measures ANOVA with phenotype (ST and GT) as between-subjects factor. The same factor and drug were utilized for the analysis of drug effects in both groups. In the PPI experiments, ANOVA included the range of the prepulse stimulus as a factor and a Student $t$-test to analyze startle amplitude. Similar analyses were conducted in the experiment with NVHL animals, including the lesion statues as a factor. All post hoc effects were analyzed with a Tukey test. 
a

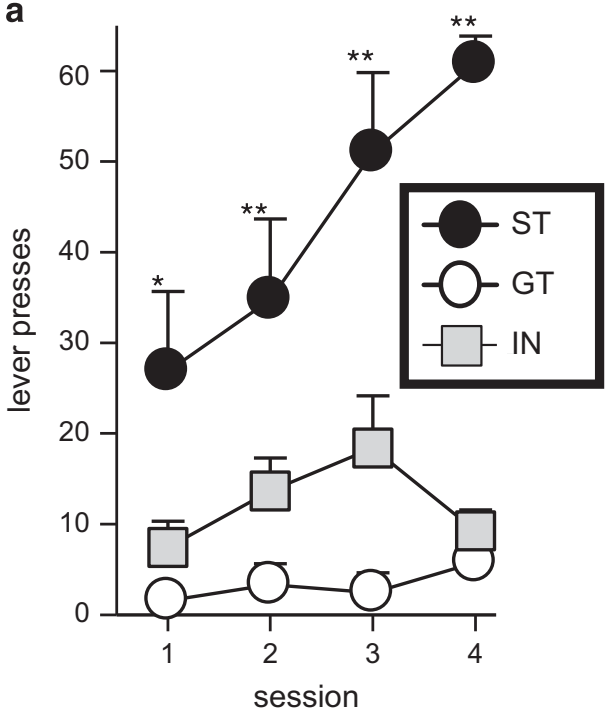

C

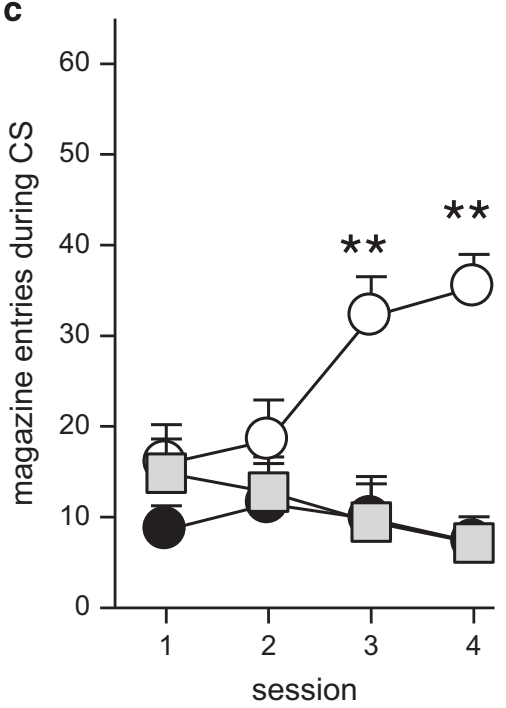

b

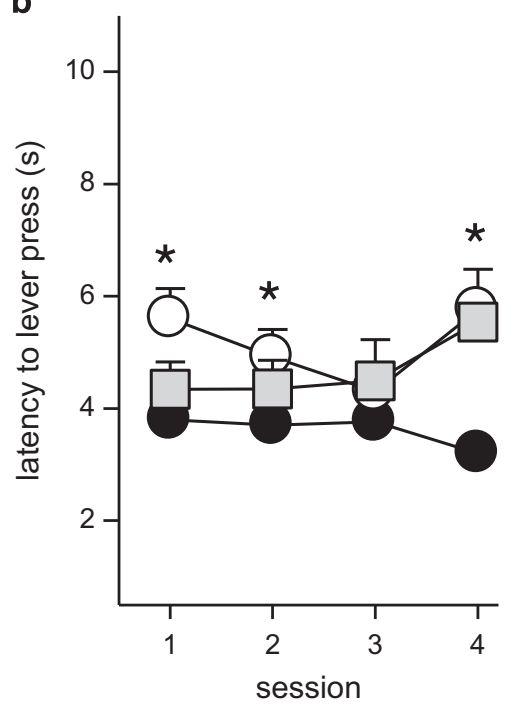

d

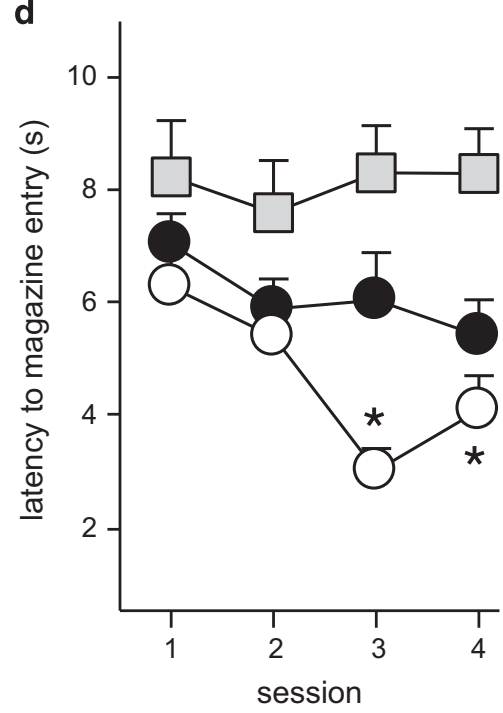

Figure I Performance in sign trackers ( $n=9$; black circles), goal trackers ( $n=9$; white circles), and intermediate groups ( $n=27$; gray squares). (a) Sign trackers pressed the lever during CS presentation more frequently than goal trackers and showed a steady increase in lever presses across sessions. The symbols (open circles for GT, black circles for ST, and gray squares for IN) apply to all panels in the figure. (b) Plot illustrating latencies to lever press across sessions in all three groups. There was no difference among groups. (c) Goal trackers showed a significant increase in average magazine entries over sessions. (d) Plot illustrating latencies to magazine entry across sessions in all groups. Goal trackers showed a faster magazine in the later sessions compared with sign trackers. The IN group did not show a bias towards approaching levers or magazines. In this and subsequent figures, error bars represent SEM. Asterisk indicates significant differences between ST and GT performance; $*<0.05$; $* * 0.01$.

\section{RESULTS}

After four sessions of Pavlovian conditioning, 9 rats were classified as GT and 9 as ST, whereas 27 rats were not included because they did not qualify for either category. The latter were classified as the intermediate group. During the $8 \mathrm{~s}$ of CS presentation, ST rats pressed the levers (CS) more frequently than GT rats $\left(\mathrm{F}_{(3,48)}=6.25 ; p=0.001\right.$; Figure $\left.1 \mathrm{a}\right)$. ST rats increased their lever pressing across sessions $\left(\mathrm{F}_{(3,24)}=7.98 ; p=0.001 ;\right.$ Figure 1a), but lever pressing remained constant across all training sessions in GT rats. There were no significant between-session differences in the latency scores $\left(\mathrm{F}_{(3,24)}=0.54 ; p=0.65\right.$; Figure $\left.1 \mathrm{~b}\right)$. In contrast to ST rats, GT rats showed a behavior oriented to the search of reward. These animals displayed low lever-pressing behavior and high frequency of magazine entries. A repeated measures ANOVA revealed an increase of magazine entries when the CS was present across sessions $\left(\mathrm{F}_{(3,24)}=8.55\right.$; $p<0.001$; Figure 1c), and the interaction confirmed that GT rats exhibited higher scores than ST across training sessions $\left(\mathrm{F}_{(3,48)}=6.22 ; p=0.001\right)$. The latencies from CS presentation to magazine entries decreased with subsequent sessions in the GT group $\left(\mathrm{F}_{(3,24)}=5.47 ; p=0.005\right.$, Figure $\left.1 \mathrm{~d}\right)$. Intermediate group rats did not show differences across sessions in lever press $\left(\mathrm{F}_{(3,72)}=2.19 ; p=0.096\right.$, Figure 1a), nose feeder responses $\left(\mathrm{F}_{(3,72)}=1.98 ; p=0.12\right.$, Figure $\left.1 \mathrm{c}\right)$, or latencies 
a

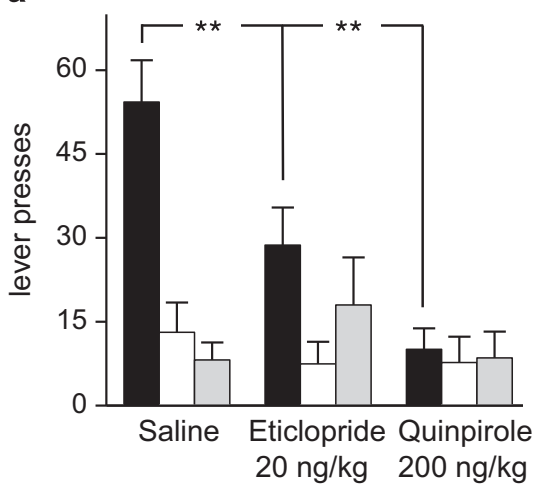

b

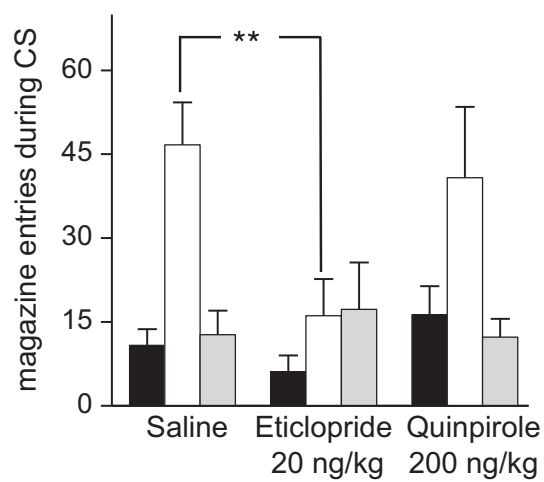

C

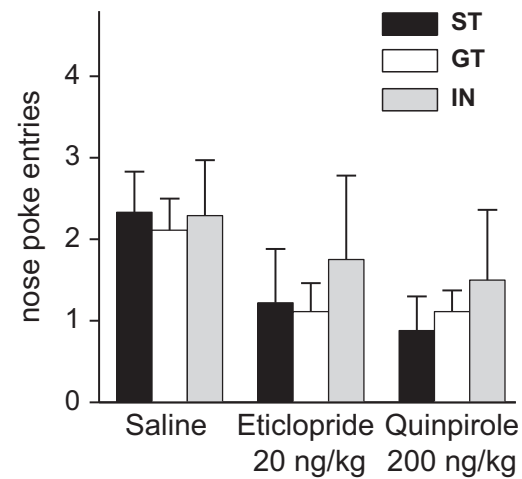

Figure 2 D2 modulation of responses in sign and goal trackers. Data show mean of lever press and magazine entries response in GT, ST, and IN groups. (a) Both eticlopride $(20 \mathrm{ng} / \mathrm{kg})$ and quinpirole $(200 \mathrm{ng} / \mathrm{kg})$ decreased lever press responding in ST rats, with the D2 agonist quinpirole showing a larger attenuation compared with saline-treated rats. (b) The D2 antagonist eticlopride attenuated the responding in GT rats whereas quinpirole administration had no effect on GT behavior. (c) D2 drugs affected nose poke activity in both groups in a similar manner $\left(F_{(1,16)}=0.009 ; p=0.92\right)$. *** $<<0.01$.

$\left(\mathrm{F}_{(3,72)}=0.742 ; p=0.53\right)$. Exploratory activity was measured with the number of entries to the nose-poke detector, and there were no differences in nose-poke entries between ST and GT rats across sessions $\left(\mathrm{F}_{(3,48)}=0.75 ; p=0.73\right)$. However, both groups showed a decrease in the number of nose-poke entries with subsequent sessions $\left(\mathrm{F}_{(3,48)}=6.41\right.$; $p=0.001$, data not shown). As the weight range of rats included was high, we assessed whether weight correlated with performance. No significant correlation was observed between the weight of each rat and its performance (ST Pearson's $r$ between 0.184 and 0.441 and GT between 0.39 and 0.59; all $p>0.92$ ). Thus, we obtained a reliable distinction between GT and ST behaviors in our rat cohorts.

\section{D2 Agonist and Antagonist Affect ST Behavior}

D2 drugs affected both GT and ST behaviors. A main effect was found in the D2 modulation of lever-pressing behavior in ST rats $\left(\mathrm{F}_{(2,16)}=9.45 ; p=0.001\right.$; Figure $\left.2 \mathrm{a}\right)$. Post hoc analyses (Tukey) revealed that both the agonist quinpirole and the antagonist eticlopride significantly reduced lever press activity (both $p<0.01$ ). While ST rats showed normal activity under saline injection, similar to training trials, eticlopride decreased performance by $50 \%$ relative to saline injection (Figure 2a). Quinpirole had a stronger effect, reducing lever pressing in ST rats by an even higher margin (Figure 2a). Whereas there was no difference in magazine entries in the ST group $\left(\mathrm{F}_{(2,24)}=2.18 ; p=0.13\right.$; Figure $\left.2 \mathrm{~b}\right)$, there was a significant effect of drug in $\mathrm{GT}$ rats $\left(\mathrm{F}_{(2,16)}=5.23\right.$; $p=0.018$; Figure 2b). The number of magazine entries in GT rats decreased with eticlopride $(p=0.008)$, but not quinpirole $(p=0.46)$. D2 drugs affected exploration in ST rats; we observed a main drug effect $\left(\mathrm{F}_{(2,32)}=7.9 ; p=0.017\right.$, Figure $\left.2 \mathrm{c}\right)$, but neither a group effect $\left(\mathrm{F}_{(1,16)}=0.009 ; p=0.92\right)$ nor an interaction $\left(\mathrm{F}_{(2,32)}=0.241 ; p=0.86\right)$. Intermediate group rats did not show differences in the response (lever press or magazine entries) for any drug treatment (saline, quinpirole, or eticlopride) $\left(\mathrm{F}_{(2,48)}=2.58 ; p=0.086\right)$. The results for GT and ST rats are summarized in Table 1. The data indicate that although D2 receptor blockade affects both GT and ST behaviors, activating D2 receptors impairs only ST behavior.
Table I Summary of Changes in Lever Press and Magazine Entries Induced by the D2 Antagonist Eticlopride $(20 \mathrm{ng} / \mathrm{kg})$ and the D2 Agonist Quinpirole (200 ng/kg)

\begin{tabular}{lcccccc}
\hline & \multicolumn{2}{c}{ Eticlopride $20 \mathbf{n g} / \mathbf{k g}$} & & \multicolumn{2}{c}{ Quinpirole } & $200 \mathbf{n g} / \mathbf{k g}$ \\
\cline { 2 - 3 } \cline { 5 - 6 } \cline { 5 - 6 } & ST & GT & & ST & GT \\
\hline Lever press & $\downarrow$ & $?$ & & $\downarrow \downarrow$ & $?$ \\
Magazine entries & $?$ & $\downarrow \downarrow$ & & $?$ & No effect \\
\hline
\end{tabular}

Arrows show the direction of the changes in both groups. Question marks indicate behavioral endpoints with low values in both groups, with uncertain effect of dopamine due to a possible floor effect.

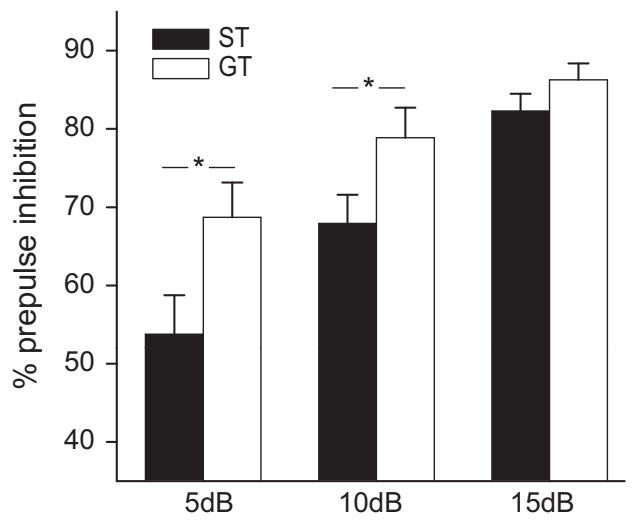

Figure 3 Reduced prepulse inhibition of the acoustic startle response in ST $(n=1 \mathrm{I})$ rats. Compared with GT $(n=1 \mathrm{I})$, ST had a significantly reduced prepulse inhibition of startle at 5 and $10 \mathrm{~dB}$ above background noise, but not at $15 \mathrm{~dB}$. $* p<0.05$.

\section{PPI in GT and ST Rats}

A different cohort of 11 ST and 11 GT rats was tested for PPI after four autoshaping sessions (Figure 3). Repeated measures ANOVA showed a significant effect of prepulse intensity $\left(\mathrm{F}_{(2,40)}=53.37, p=0.01\right)$, a main effect of group 


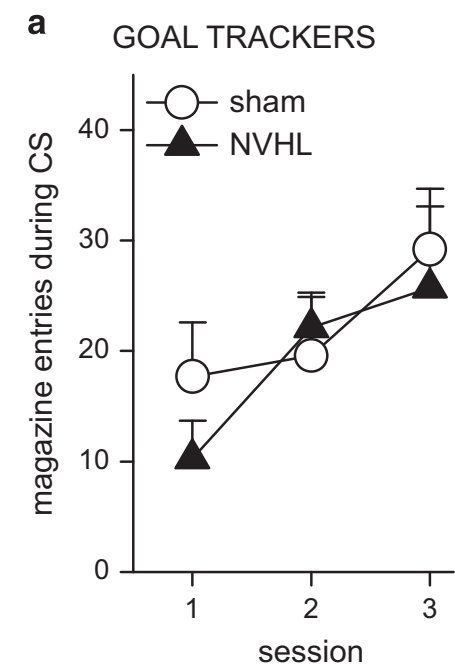

b

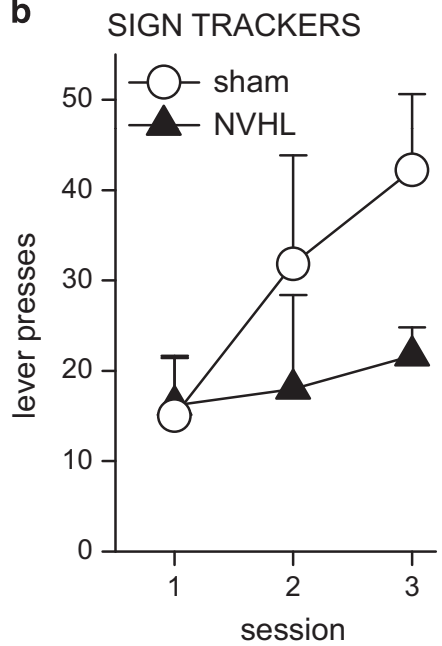

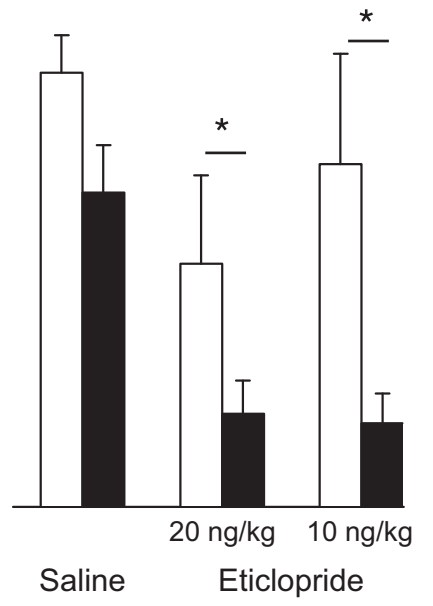

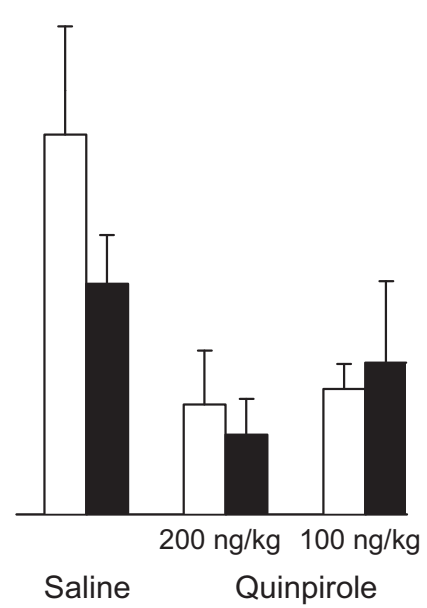

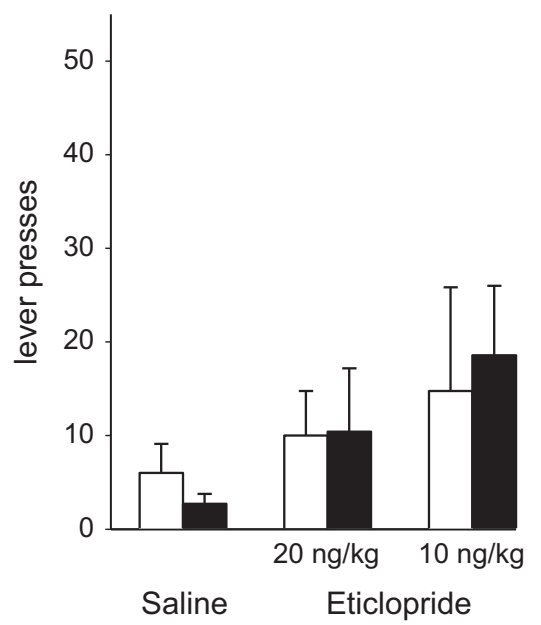

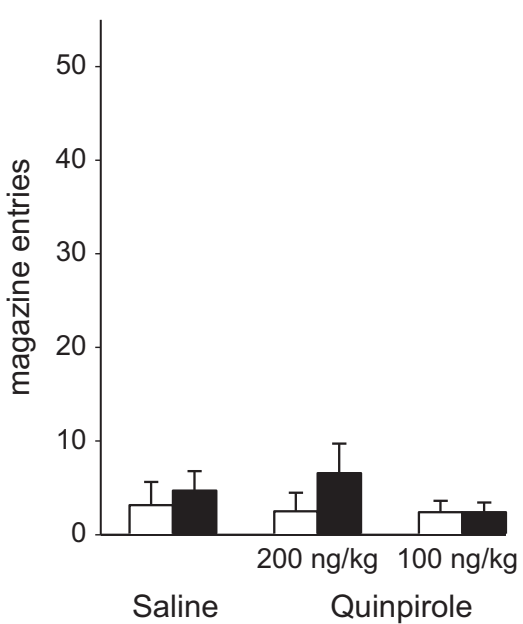

Figure 4 D2 modulation has a greater effect in GT NVHL rats $(n=7)$. (a, left) NVHL rats showed similar levels of magazine entries as sham controls ( $n=7$ ). Center: both doses of eticlopride ( $10 \mathrm{ng} / \mathrm{kg}$ and $20 \mathrm{ng} / \mathrm{kg}$ ) decreased responding in NVHL rats, but only higher doses of eticlopride decreased the response in sham animals. Right: lever press response did not show any variation. (b) Sham $(n=7)$ and NVHL $(n=6)$ ST rats showed a different response pattern: while sham rats increased lever pressing across sessions, NVHL rats did not (left). Center: quinpirole administration decreased the response in both groups. Right: magazine response was similar in both groups. Data show mean of lever press and magazine entries response. ${ }^{*} p<0.05$.

$\left(\mathrm{F}_{(1,20)}=5.11 ; p=0.035\right)$, and a significant interaction between prepulse intensity and group $\left(\mathrm{F}_{(2,40)}=3.21, p=0.05\right)$. Post hoc analysis (Tukey) revealed a significantly reduced PPI at the low- and mid-intensity prepulses in ST rats compared with GT rats $\left(5 \mathrm{~dB}\right.$ above background: $t_{(20)}=2.23, p=0.037$; $\left.10 \mathrm{~dB}: t_{(20)}=2.26 ; p=0.035\right)$, but no differences in the high-intensity $\left(15 \mathrm{~dB} ; t_{(20)}=1.30 ; p=0.20\right)$. Finally, ST and GT groups did not differ significantly in startle amplitude $\left(t_{(20)}=0.92 ; p=0.36\right.$, data not shown). Thus, PPI, an assay sensitive to dopamine, revealed differences between ST and GT rats.

\section{ST and GT Behavior in NVHL Rats}

In a separate group of rats, we tested whether adult rats with a NVHL $(n=13)$ or sham-operated rats $(n=14)$ showed differences in GT and ST behaviors. Eight NVHL and nine sham animals were excluded because they did qualify for either behavioral profile. GT rats increased the number of magazine entries across sessions $\left(\mathrm{F}_{(2,24)}=14.57 ; p=0.001\right.$; Figure $4 \mathrm{a})$ at a similar rate $\left(\mathrm{F}_{(2,24)}=1.74 ; p=0.19\right)$, regardless of lesion status. D2 antagonist administration had a different effect on magazine entries in NVHL $v s$ sham rats $\left(\mathrm{F}_{(2,24)}=3.7 ; p=0.04\right.$, Figure $\left.4 \mathrm{a}\right)$, but there were no differences in lever-directed behavior (Figure $4 \mathrm{a}$ ). While $20 \mathrm{ng} / \mathrm{kg}$ eticlopride had a similar effect in either group $(p=0.13)$, a lower dose $(10 \mathrm{ng} / \mathrm{kg})$ reduced magazine entries in NVHL but not sham rats $(p=0.018)$. Thus, GT strategy is more sensitive to D2 modulation in NVHL rats than in controls. Lever press performance remained constant in each test $\left(\mathrm{F}_{(2,24)}=.58 ; p=0.56\right.$, Figure $\left.4 \mathrm{a}\right)$.

Performance of ST rats, on the other hand, was different in sham and NVHL groups. Whereas sham rats increased their lever pressing through consecutive sessions $\left(\mathrm{F}_{(2,12)}=4.11\right.$; $p=0.04$; Figure $4 \mathrm{~b}$ ), NVHL rats did not change their performance $\left(\mathrm{F}_{(2,10)}=0.4 ; p=0.68\right)$, showing a high but stable response rate and lower scores than sham rats. On the other hand, pharmacological data did not show any differences 
between groups $\left(\mathrm{F}_{(2,22)}=0.7 ; p=0.5\right.$; Figure $\left.4 \mathrm{~b}\right)$. There were no differences in magazine-directed behavior between NVHL and sham ST rats (Figure 4b). To rule out the possibility that a different motor activity level would cause a reduced performance in NVHL rats, we analyzed responses to a nosepoke detector in ST and GT rats. Nose-poke activity was similar during all sessions and no statistical differences between sham and NVHL animals were found $\left(\mathrm{F}_{(9,69)}=0.44\right.$; $p=0.90)$. Combining the observations in NVHL rats, this manipulation yields adult rats with a weaker ST approach and an increased frequency of GT behavior, which unlike in control animals, is more sensitive to D2 receptor blockade.

\section{DISCUSSION}

GT and ST rats exhibited differences in their behavior when corticolimbic dopamine transmission was manipulated. First, D2 agonist and antagonist administration differently affected the performance of ST and GT rats. Although a D2 antagonist impaired performance in rats with either profile, a D2 agonist impaired performance only in ST rats. Second, ST rats showed lower PPI than GT rats, revealing poorer sensorimotor gating in a task that requires dopamine receptor activation (Swerdlow et al, 2001; Braff, 2010). Third, rats with a NVHL, a developmental manipulation that alters corticolimbic dopamine functions, exhibited altered responses in ST rats, which performed poorer than sham controls, and increased sensibility to D2 blockade in GT rats.

The different D2 receptor modulation in ST vs GT rats may relate to how reward is processed in each behavioral strategy. Corticolimbic dopamine neurotransmission is related to reward-prediction error (Roesch et al, 2012; Steinberg et al, 2013), and mesolimbic dopamine neurons are likely activated in anticipation of reward by the CS, depending on whether reward is expected or not (de la Fuente-Fernandez et al, 2002; Ito et al, 2002; Phillips et al, 2003; Tobler et al, 2005; Roesch et al, 2012). These observations have been interpreted as dopamine acting as a teaching signal for predicting errors (Berridge, 2007). Incentive salience and error prediction are two proposed functions of dopamine that are difficult to assess in an independent way. Saunders and Robinson (2012) found support for the involvement of dopamine in incentive salience to reward cues. In contrast, Steinberg et al (2013) observed some differences in dopamine neuron activity during reward delivery in a blocking and extinction procedure, as a support to prediction error models. Our data indicate that dopamine has a role in the incentive salience of a discrete stimulus. A D2 agonist decreased lever pressing in ST rats, but not in GT rats, when the prediction error was zero. This observation could be explained by D2 activation of autoreceptors in dopamine neurons, which would reduce activity levels in these cells and hamper the processing of salient signals. On the other hand, GT performance was considerably reduced with the administration of the D2 antagonist eticlopride. These data are consistent with those observed with the D1-D2 antagonist flupenthixol in rats with a high (bHR) and low (bLR) responding profile to novelty (Flagel et al, 2011). In that study, dopamine reduced incentive salience of the CS during acquisition of a ST conditioned reward in ST rats, but not GT rats, but reduced performance in both groups. In addition,
Saunders and Robinson (2012) reported a selective role of dopamine in the NA core in the performance of ST rats. Dopamine blockade in NA decreased the conditioned reward once the response had been learned. This finding attributes dopamine in the NA with a central role in learned responses, but only in ST rats. Our data extends these findings, showing that the dopamine system involved in these responses is more complex. We observed that a D2 agonist reduced the number of contacts to a greater extent than what were caused by eticlopride. This paradoxical result, observed only in ST rats, could be the reflection of engagement of multiple locations of D2 receptors within ventral striatal circuits (ie, postsynaptic in D2-expressing medium spiny neurons, presynaptic in dopamine and glutamate afferents, in striatal interneurons, etc.) and/or engagement of extrastriatal D2 receptors (ie, in the prefrontal cortex). Thus, when processing of salient cues is critical, an optimal tone of dopamine and D2 receptor activation would be required for a suitable performance in these circuits.

ST and GT rats also differed in dopamine-modulated attentional processes, such as sensorimotor gating. ST rats showed reduced PPI compared with GT rats. The data could be interpreted as enhanced PPI in GT rats or impaired PPI in ST rats. Dopamine in the NA is a critical element in the attenuation of the startle response to an intense pulse when it is preceded by a lower-intensity prepulse stimulus (Swerdlow et al, 2001). The CS elicits a rapid rise in NA dopamine in ST rats (Flagel et al, 2011; Schultz, 2013), and this could yield a lower PPI response, suggesting a reduced inhibitory ability in the behavioral pattern. As similar results have been observed under effects of drugs that increase dopamine release in the NA, a reduced PPI suggests that sensorimotor gating may be poorer in ST subjects.

ST and GT behavior was also differently expressed and differently modulated by dopamine in a developmental manipulation that yields adult animals with altered dopamine modulation of corticostriatal circuits. The NVHL models adolescent onset of cognitive deficits in adult rats (O'Donnell, 2011). The impaired hippocampal innervation at a critical developmental period may alter developmental trajectories of prefrontal-striatal synaptic connectivity and function (O'Donnell et al, 2002; Tseng et al, 2008; O'Donnell, 2011,2012). Altered corticostriatal activity and its dopamine modulation could affect incentive salience. This hypothesis is consistent with the findings that activation of the ventral hippocampus is necessary to increase dopamine release throughout the NA (Legault and Wise, 1999; Floresco et al, 2001). The NVHL could blunt the elevation of NA dopamine when the CS was present, and this might explain the low ST performance across sessions. On the other hand, the NVHL could facilitate GT performance by reducing the CS incentive salience. However, pharmacological data show vulnerability in GT animals to dopamine manipulations. Low doses of eticlopride affected the performance in NVHL, but not sham rats, suggesting that the lesion renders adult animals with increased vulnerability to behavioral effects of dopamine pharmacology.

In conclusion, ST and GT rats displayed different traits associated with behavioral disinhibition (Flagel et al, 2007; Robinson and Flagel, 2009; Flagel et al, 2011). The different D2 modulation of GT and ST responses suggests the possibility of impulsive behavior being a trait of ST rats. 
Indeed, ST rats have been reported as showing propensity for impulsive action (Lovic et al, 2011), and D2 receptors could play a role in the modulation of this trait. The reduced PPI in ST rats further suggests a different processing of salient sensory stimuli in this group. Finally, a developmental deficit in corticolimbic dopamine functions affected both phenotypes in different ways, underscoring the complexity of neural systems associating cues with rewards. Overall, our data support the possibility to use ST behavior as a model of cue-induced impulsivity, because impulsive behavior is significantly reduced by D2 in NA (Flagel et al, 2010; Dalley and Roiser, 2012).

\section{FUNDING AND DISCLOSURE}

This research was supported by PR2010-0035 and PSI201232445 grants (JCL) and NIH grant R01 MH57683 (POD). Patricio O'Donnell is currently employee and stockholder at Pfizer, Inc. The authors declare no conflict of interest.

\section{REFERENCES}

Berridge KC (2007). The debate over dopamine's role in reward: the case for incentive salience. Psychopharmacology 191: 391-431.

Berridge KC, Robinson TE (1998). What is the role of dopamine in reward: hedonic impact, reward learning, or incentive salience? Brain Res Rev 28: 309-369.

Braff DL (2010). Prepulse inhibition of the startle reflex: a window on the brain in schizophrenia. Curr Top Behav Neurosci 4: 349-371.

Chambers RA, Lipska BK (2011). A method to the madness: producing the neonatal ventral hippocampal lesion rat model of schizophrenia. In: O’Donnell P (ed) Animal Models of Schizophrenia and Related Disorders. Humana Press: New York. pp 1-24.

Dalley JW, Roiser JP (2012). Dopamine, serotonin and impulsivity. Neuroscience 215: 42-58.

de la Fuente-Fernandez R, Phillips AG, Zamburlini M, Sossi V, Calne DB, Ruth TJ et al (2002). Dopamine release in human ventral striatum and expectation of reward. Behav Brain Res 136: 359-363.

Flagel SB, Clark JJ, Robinson TE, Mayo L, Czuj A, Willuhn I et al (2011). A selective role for dopamine in stimulus-reward learning. Nature 469: 53-57.

Flagel SB, Robinson TE, Clark JJ, Clinton SM, Watson SJ, Seeman P et al (2010). An animal model of genetic vulnerability to behavioral disinhibition and responsiveness to reward-related cues: implications for addiction. Neuropsychopharmacology 35: 388-400.

Flagel SB, Watson SJ, Robinson TE, Akil H (2007). Individual differences in the propensity to approach signals vs goals promote different adaptations in the dopamine system of rats. Psychopharmacology 191: 599-607.
Floresco SB, Todd CL, Grace AA (2001). Glutamatergic afferents from the hippocampus to the nucleus accumbens regulate activity of ventral tegmental area dopamine neurons. J Neurosci 21: 4915-4922.

Goto Y, O'Donnell P (2002). Delayed mesolimbic system alteration in a developmental animal model of schizophrenia. J Neurosci 22: 9070-9077.

Ito R, Dalley JW, Robbins TW, Everitt BJ (2002). Dopamine release in the dorsal striatum during cocaine-seeking behavior under the control of a drug-associated cue. J Neurosci 22: 6247-6253.

Legault M, Wise RA (1999). Injections of N-methyl-D-aspartate into the ventral hippocampus increase extracellular dopamine in the ventral tegmental area and nucleus accumbens. Synapse 31: 241-249.

Lovic V, Saunders BT, Yager LM, Robinson TE (2011). Rats prone to attribute incentive salience to reward cues are also prone to impulsive action. Behav Brain Res 223: 255-261.

Meyer PJ, Lovic V, Saunders BT, Yager LM, Flagel SB, Morrow JD et al (2012). Quantifying individual variation in the propensity to attribute incentive salience to reward cues. PloS One 7: e38987.

O'Donnell P (2011). Adolescent onset of cortical disinhibition in schizophrenia: insights from animal models. Schizophr Bull 37: 484-492.

O'Donnell P (2012). Cortical disinhibition in the neonatal ventral hippocampal lesion model of schizophrenia: New vistas on possible therapeutic approaches. Pharmacol Ther 133: 19-25.

O'Donnell P, Lewis BL, Weinberger DR, Lipska BK (2002). Neonatal hippocampal damage alters electrophysiological properties of prefrontal cortical neurons in adult rats. Cereb Cortex 12: 975-982.

Phillips PE, Stuber GD, Heien ML, Wightman RM, Carelli RM (2003). Subsecond dopamine release promotes cocaine seeking. Nature 422: 614-618.

Robinson TE, Flagel SB (2009). Dissociating the predictive and incentive motivational properties of reward-related cues through the study of individual differences. Biol Psychiatry 65: 869-873.

Roesch MR, Esber GR, Li J, Daw ND, Schoenbaum G (2012). Surprise! Neural correlates of Pearce-Hall and Rescorla-Wagner coexist within the brain. Eur J Neurosci 35: 1190-1200.

Saunders BT, Robinson TE (2012). The role of dopamine in the accumbens core in the expression of Pavlovian-conditioned responses. Eur J Neurosci 36: 2521-2532.

Schultz W (2013). Updating dopamine reward signals. Curr Opin Neurobiol 23: 229-238.

Steinberg EE, Keiflin R, Boivin JR, Witten IB, Deisseroth K, Janak PH (2013). A causal link between prediction errors, dopamine neurons and learning. Nat Neurosci 16: 966-973.

Swerdlow NR, Geyer MA, Braff DL (2001). Neural circuit regulation of prepulse inhibition of startle in the rat: current knowledge and future challenges. Psychopharmacology 156: 194-215.

Tobler PN, Fiorillo CD, Schultz W (2005). Adaptive coding of reward value by dopamine neurons. Science 307: 1642-1645.

Tseng KY, Lewis BL, Hashimoto T, Sesack SR, Kloc M, Lewis DA et al (2008). A neonatal ventral hippocampal lesion causes functional deficits in adult prefrontal cortical interneurons. J Neurosci 28: 12691-12699. 